\title{
Documentando lo intangible, las voces de la ciudad en los Barrios San Nicolás, Cali y Del Carmen, Ciudad de México
}

\author{
Margarita Cuellar Barona ${ }^{1}$, Jimena de Gortari Ludlow ${ }^{2}$ \\ ${ }^{1}$ Departamento de Artes y Humanidades, Universidad Icesi, Cali, Colombia, ${ }^{2}$ Departamento de \\ Arquitectura, Urbanismo e Ingeniería Civil, Universidad Iberoamericana Ciudad de México, México \\ E-mail: ${ }^{1}$ mlcuellar@icesi.edu.co, ${ }^{2}$ jimena.dgortari@ibero.mx,
}

\begin{abstract}
Resumen. La experiencia sensorial tuvo un papel importante en el análisis de la ciudad en el mundo pre moderno. No obstante, la progresiva racionalización del conocimiento trajo consigo el dominio de la visión sobre otros sentidos convirtiéndonos en una sociedad oculocentrista. Sabemos que la ciudad no se vive de igual manera, por tanto debemos considerar la experiencia sensorial como parte fundamental en la construcción de la memoria del lugar e incorporarla como una capa más en los estudios de la forma urbana.

A través del estudio comparativo de la experiencia de escucha de dos barrios latinoamericanos (San Nicolás en Cali, Colombia y Coyoacán en Ciudad de México) este trabajo pretende poner de manifiesto el papel que juega el sonido en la conformación de los espacios sociales urbanos, en el impacto sobre la calidad de vida y en las representaciones mentales que los y las habitantes de las ciudades construyen de sus entornos. El texto presenta una metodología de trabajo en la que se apela a la memoria, al relato, al recuerdo, a la referencia, al vínculo de lo tangible con lo intangible; a esa(s) ciudad(es) que no se puede(n) entender sin analizar la relación que existe entre las personas que habitan el espacio y el espacio en sí, del espacio como anhelo y como experiencia; en donde el sonido cobra un significado relevante y que al documentarse permitirá entender el vínculo que tienen los habitantes con el espacio público.
\end{abstract}

Palabras clave: Sonido, apropiación, memoria, experiencia, habitar.

\section{Introducción}

Los sonidos son las expresiones acústicas de la vida a medida que se despliega; son manifestaciones sonoras de la dinámica de la vida urbana. Abordar el análisis de la ciudad a través de los sonidos puede ofrecer una alternativa extremadamente útil a las metodologías orientadas a la visión que han caracterizado los estudios urbanos, nos puede ayudar a transgredir los análisis contemplativos (estáticos) que han predominado los enfoques (visuales) de los paisajes urbanos (Carles \& Palmese, 2009). Sin embargo, el sonido se ha convertido en sinónimo de ruido $\mathrm{y}$, a menudo, las palabras se intercambian al referirse al espacio público. Al igual que "basura, que públicamente llama a algo ruido (o ruidoso) lo condena implícitamente y, de manera crucial, refuerza los límites de las clasificaciones apreciadas que han sido confundidas $o$ ignoradas" (Rice, 2008). El sonido, en la vida de la ciudad, casi siempre se considera una molestia, como algo que necesita ser tratado, corregido, reducido o "eliminado". Durante la última década, el aumento en el tráfico de la ciudad ha provocado un crecimiento en las investigaciones que exploran los sonidos y los efectos negativos que tienen sobre los habitantes de la ciudad. Sin embargo, pensar en el sonido como una molestia pública está reduciendo toda la información acústica en los paisajes 
urbanos a la categoría de ruido, empobreciendo así las posibilidades comunicativas del sonido. A finales de la década de los sesenta, el compositor y académico canadiense Murray Schafer se embarcó en un proyecto para registrar los sonidos de su ciudad (Vancouver) e instó a otras personas a documentar sus propios entornos cambiantes como un medio para revisar la relación entre los sonidos, los seres humanos, la naturaleza y la sociedad. Muchos otros académicos, como Barry Truax, Hildergard Westerkamp, Bernie Krausse, se unieron a Schafer en la búsqueda de grabar y analizar sonidos para documentar sus cambiantes entornos acústicos y prevenir la degradación futura de los territorios naturales y urbanos. Gran parte de la investigación llevada a cabo en los primeros años de The World Soundscape Project tuvo como objetivo crear conciencia de la importancia de los sonidos para crear hábitats de vida saludables, dando paso a un nuevo campo de estudio llamado Ecología Acústica (Cuéllar y Llorca, 2017). Unos años más tarde (1972), en el Congreso de Medio Ambiente organizado por las Naciones Unidas en Estocolmo, el ruido fue reconocido por primera vez como un agente contaminante. Schafer introdujo una nueva forma de entender el sonido y abogó por que fuera comprendido como una característica de una comunidad determinada, de la misma manera que consideramos el paisaje o cualquier otra forma de expresión cultural. Entendemos el paisaje sonoro como un equivalente auditivo al paisaje; una porción de territorio que se puede escuchar en un momento dado en un lugar determinado, reconociendo su valor estético. Como sugirió "Barry Truax (2001), los paisajes sonoros son el resultado de encontrarse, escuchar, dar significado, responder e interactuar simultáneamente" (Duffy y Waitt, 2010) con los estímulos acústicos que nos rodean. Sin embargo, con "entorno acústico" o "entorno sonoro" nos referimos a las características sonoras de un área determinada basadas únicamente en mediciones físicas, ya que la percepción juega un papel muy importante en la forma en que experimentamos tales sonidos, lo cual es precisamente la razón por la que discutir el ruido, como categoría, es tan vago. El ruido, tal como lo define Schafer es "cualquier sonido no deseado". Sin embargo, su definición no solo es muy amplia sino que permite una interpretación muy subjetiva: si bien un sonido determinado puede considerarse ruido para algunas personas, puede pasar inadvertido para un grupo diferente $\mathrm{y}$, al mismo tiempo, ser disfrutado por otros. Entonces, si bien su preocupación por el diseño de ambientes sonoros saludables es de gran relevancia y debe ser tomada en cuenta por los planificadores urbanos y los diseñadores urbanos (precisamente el propósito de la ecología acústica), contribuye muy poco a la comprensión del sonido en relación con las prácticas sociales que tienen lugar. en un entorno dado, particularmente en el de un contexto urbano.

Actualmente, la Organización Mundial de la Salud (OMS) estima que el ruido es la segunda causa de enfermedades originadas por el medio ambiente, justo por debajo de la contaminación atmosférica. La exposición al ruido ha aumentado en los últimos años, ya que se ha "extendido desde los centros urbanos a las áreas suburbanas y rurales y desde el día hasta la noche, reduciendo así las opciones viables para" (Brooks, et al., 2014) descanso y tranquilidad, ocasionando un deterioro de la calidad de vida.

'While classical noise indicators are known to show strong limitations under certain sound conditions (low frequency noise, tonal components, multisource environments), it is central to soundscape research and the implementation of fitting the applied indicators to perception and appraisal of those concerned. The fitted indicators also depend, however, on the type of the soundscape investigated. It is extremely important that the fitting of indicators reflects the situation and context (personal, social, cultural, land use, economic, geographic) which define the sonic listening space, and also enables tracing dynamic changes like time variations of the soundscape over the day or seasons.' (Brooks, 2010)

Como se indica en el artículo de Brooks (2010) al explorar nuestro entorno sonoro la evaluación no debe depender únicamente de las características físicas de sus sonidos (intensidad y frecuencia) sino de la información que puedan proporcionar con respecto al 
contexto en el que ocurren, cómo se perciben, y los significados culturales y sociales que transmiten (López, et.al, 1997, Dubois, et.al, 1999). Los paisajes sonoros deben considerarse, no solo desde una mera evaluación acústica o desde el punto de vista del control del ruido, sino como la representación de la interacción entre los seres humanos (y otras especies), con el entorno que habitan (Brown, 2010). Los paisajes sonoros urbanos son producto de una gran combinación de sonidos que, como en una pieza musical, se mezclan para formar una sinfonía, o quizás (para ser más precisos), una cacofonía que no tiene fin. No necesariamente somos conscientes de todos los matices en tales composiciones y tendemos a hacer una audición selectiva, identificando los sonidos que conocemos, aquellos que nos transmiten un mensaje, nos alertan o contienen ciertos significados. La investigación en el campo debe combinar las mediciones del entorno sónico con información sobre experiencias auditivas para evaluar adecuadamente cómo se relacionan los individuos con el sonido, especialmente en los hábitats urbanos (Ge, Jian; Hokao, Kazunori, 2005). Los estudios sobre el sonido no deben centrarse exclusivamente en preservar, alentar o multiplicar los que se encuentran agradables, ni eliminar los que se consideran desagradables, sino como un medio para estudiar el tiempo y el lugar. Las ciudades están ocupadas, llenas de gente y en constante movimiento, por lo tanto, debemos ir más allá de pensar en el sonido como algo que altera la calidad de vida en la ciudad y avanzar hacia considerarlo como una expresión de eso. Reconocer que el ruido (o la abundancia de sonidos) es un elemento esencial de la vida en la ciudad es el primer paso para repensar el sonido como una molestia o una simple señal para ser procesada, pero como información digna de ser analizada.

Este documento tiene como objetivo abordar el estudio de la vida en la ciudad a través de sus sonidos, comparando la experiencia auditiva de dos barrios ruidosos diferentes: Coyoacán, Ciudad de México y San Nicolás, Cali, Colombia. Nuestra investigación está dirigida a construir cartografías audibles (o mapas sonoros) como un medio para combinar un análisis visual de los vecindarios y el sonido, a fin de examinar su impacto en la vivienda de la ciudad. Este documento discute las metodologías utilizadas para construir tales cartografias y propone que, escuchar atentamente el sonido (o mejor dicho, el ruido) ayudará a dar forma a la comprensión de la vida cotidiana en la ciudad, el efecto que el sonido tiene sobre el uso espacial (público), el impacto que tiene sobre la calidad de vida y cómo da forma a las representaciones mentales que los habitantes de las ciudades construyen del entorno urbano. Nuestro objetivo es proporcionar modos de análisis que puedan ayudarnos a abordar múltiples voces en la ciudad, reflexionando sobre la calidad de vida y como características de la vida urbana.

\section{Escuchando la ciudad}

Now I will do nothing but listen,

To accrue what I hear into this song, to let sounds contribute toward it.

I hear bravuras of birds, bustle of growing wheat, gossip of flames,

clack of sticks cooking my meals,

I hear the sound I love, the sound of the human voice,

I hear all sounds running together, combined, fused or following,

Sounds of the city and sounds out of the city, sounds of the day and night...

(Song of Myself, Walt Whitman)

Jane Jacobs (1961) sostiene que los espacios públicos son las arenas en las que se desarrolla la vida en la ciudad, donde reside su vitalidad. Para analizar la dinámica de la vida urbana, es necesario comprender cómo los habitantes interactúan con los espacios públicos, los significados que asignan a dichos lugares y las actividades que realizan en ellos. Para aprender sobre esas interacciones, uno necesita ir a las calles y hablar con la gente; leer en las relaciones, enlaces y conexiones que la ciudad genera entre ellos; necesitamos escuchar a la ciudad y a sus habitantes. En otras palabras, para analizar la vida en la ciudad no es suficiente entender cómo es la ciudad, de qué está hecha, sino cómo se siente y cómo suena. La investigación actual sobre el sonido y la 
experiencia sensorial destacan la importancia de integrar metodologías que combinen información cuantitativa y cualitativa para abordar el sonido en relación con sus impactos en el medio ambiente $\mathrm{y}$, posteriormente, en la vida cotidiana. La perspectiva cuantitativa requiere el uso de instrumentos para medir las características del sonido, como la presión del sonido y las frecuencias, así como la realización de un análisis espacial exhaustivo. Los decibeles medidos en espacios urbanos dependen de las características de la fuente de sonido y de las condiciones de propagación del sonido. El comportamiento del sonido en las calles está determinado por los elementos que forman las fachadas, así como por las irregularidades en los edificios y la superficie del terreno; también debemos reconocer que los niveles de sonido dependen del ancho de la calle y la altura de los edificios, y que la textura de los recubrimientos horizontales y verticales favorece la propagación y el reflejo de la energía acústica. (De Gortari, 2013). La perspectiva cualitativa utiliza diversas metodologías (collage de diferentes disciplinas) para analizar cómo las personas perciben su entorno acústico y cómo influye en la forma en que habitan en dichos espacios.

Los investigadores que trabajan en este campo provienen de diferentes orígenes como arquitectura, biología, estudios urbanos, psicología, arte, antropología, geografía, historia, sociología, etc. (Westerkamp, 1972; Augoyard, 1997; Bull, 2000; Thibaud, 2003; Alonso, 2003, 2005; Carles \& Palmese, 2004; Amphoux (2001), Daumal (2002), Paquette, 2004; Atienza, 2008; De Gortari, 2010; Domínguez, 2012; Llorca, 2017), y han generado nuevas vías teóricas y metodológicas, que señalan la importancia de tener un enfoque holístico de la manera en que concebimos el sonido. Hasta cierto punto, la mayoría de las disciplinas mencionadas anteriormente tienen algo en común, ya que tratan con la experiencia humana, que fue el objetivo principal de nuestro proyecto: comprender cómo el sonido da forma a la forma en que las personas viven en estos dos barrios.

La investigación realizada tanto en Coyoacán como en San Nicolás, tenía la intención de crear mapas audibles a través de los cuales pudiéramos documentar el ambiente sonoro de los vecindarios, pero también mapas en los que sus habitantes pudieran reconocer los sonidos de sus alrededores y que nos ayudarán a analizar cómo afectan dichos sonidos su calidad de vida. Por lo tanto, una primera fase caracteriza ambos barrios en términos sociales y espaciales, lo que significó cuestionar su forma urbana, para ello se consultó material de archivo para identificar cambios en su configuración espacial; se consultaron mapas históricos, archivos fotográficos y libros de historia local para analizar sus transformaciones. También se completó un análisis detallado del uso de la tierra para evaluar los cambios demográficos en el vecindario y para comprender mejor su dinámica social.

El resultado de estas actividades permitió una caracterización de los vecindarios que revelaron cambios en sus usos residenciales y comerciales, impuestos por una economía fluctuante; un aumento en el turismo, en el caso de Coyoacán, y la descentralización del centro de la ciudad, en San Nicolás, pero que, sin embargo, han logrado conservar un carácter distinto, también presente en su entorno sonoro. En el caso de San Nicolás, la investigación se centró en documentar la historia de la industria de las artes gráficas en relación con el vecindario y, por lo tanto, se consultó como fuente principal los archivos de la Cámara de Comercio. En el caso de Coyoacán, se consultaron mapas históricos para comprender la transformación del área y la reconfiguración espacial de sus calles y plazas. La información se complementó con las crónicas de la ciudad que hablan de la vida en este espacio, además de fotografías históricas recopiladas de diversos archivos y textos. Toda esta información ha permitido configurar algunos mapas para comprender la transformación socio-espacial de las plazas analizadas.

La segunda fase buscó registrar, mapear y analizar el ambiente sonoro de los barrios. Esta fase tenía dos objetivos principales: primero; para caracterizar la dinámica sonora y socioespacial de ambos lugares, y segundo; para investigar cómo las estructuras de sonido son experimentadas por aquellos que habitan en estos espacios. El primer objetivo respondió a la necesidad de identificar los usos que las 
personas hacen del espacio, las dinámicas y los ritmos que surgen de tales usos, así como identificar los sonidos que se escuchan con mayor frecuencia en diferentes momentos del día. El segundo objetivo fue explorar el papel que desempeña en las representaciones mentales (individuales y colectivas) de los vecindarios y comparar cómo el sonido da forma a la forma en que los residentes y visitantes perciben el espacio.

Dado que ambos proyectos tenían como objetivo crear cartografías sonoras en los que los usuarios podían localizar lugares y fuentes donde se escuchaban los sonidos, se registraron muestras de sonido. Estas grabaciones se colocaron posteriormente en mapas digitales que atestiguan la variedad de sonidos que se pueden escuchar en ambos vecindarios.

Las metodologías utilizadas para construir mapas de sonido nos permiten desarrollar la noción de lugar en toda su complejidad. Significa escuchar y grabar la experiencia auditiva del espacio desde una posición determinada, a una hora determinada y en un día determinado. Esto crea conciencia de la importancia del sonido y su relación con nuestras percepciones del espacio y el lugar. Revela cómo un análisis más cercano de los paisajes sonoros de la ciudad puede constituir rutas alternativas para comprender las relaciones que coexisten entre los individuos y su entorno. Las cartografías sonoras son un recurso importante para los planificadores urbanos y el diseño arquitectónico al ubicar fuentes de sonido y vincularlas con las respuestas humanas. Los mapas son un lenguaje expresivo (construcción gráfica) que materializa los entornos acústicos mediante la recopilación de la escucha y el pensamiento, así como el sonido y el espacio (Ipinza, C., Hidalgo, A. 2017).

\section{San Nicolás, Cali (Colombia)}

Como la mayoría de las ciudades de América Latina, el centro de la ciudad de Cali ya no es el centro real de la ciudad. Si bien todavía tiene oficinas gubernamentales, bancos $\mathrm{y}$ notarios públicos, ya no es un área residencial y la mayoría de sus tiendas, restaurantes y panaderías cierran después de las 6:00 p.m. cuando lentamente se abandona. Como la mayoría de las ciudades de América Latina, el centro de la ciudad de Cali alberga en su mayoría economías informales y actividades comerciales de bajo nivel que se realizan únicamente durante el día, cuando las aceras se llenan de mendigos y vendedores ambulantes, que pueden vender cualquier cosa, desde boletos de lotería, frutas, verduras, etc. Marcos de gafas, ropa, zapatos, juguetes baratos que funcionan con batería, copias ilegales de CD y DVD, así como una serie de artilugios curiosos para la casa. Los centros urbanos suelen ser apestosos, ocupados, densos, intensos, atestados, peligrosos $\mathrm{y}$, sobre todo, ruidosos.

San Nicolás es uno de los barrios que conforman el centro de la ciudad (Comuna 3) y está ubicado a solo dos cuadras de la plaza más famosa de Cali: Plaza de Cayzedo. Cuando se estableció el vecindario, estos dos bloques fueron significativos, ya que hicieron la diferencia entre tener calles pavimentadas (con piedras) para tener caminos de tierra. Hoy en día, el vecindario ha sido completamente absorbido por las actividades comerciales y alberga el famoso San Andresito, un complejo comercial donde se pueden comprar productos de contrabando como licor, ropa, zapatos y electrodomésticos (equipos de música, televisores, hornos de microondas, etc.) en precios mucho más baratos. El vecindario también es conocido por la venta de altavoces y estéreos, que se anuncian al tocarlos en voz alta en la acera, compitiendo con el sonido de los vendedores ambulantes, que venden copias piratas de la última película o álbum de música, y recurren a la publicidad de su mercancía de forma descarada. Gritar los últimos títulos o reproducirlos en un televisor o un sistema de sonido, conectado ilegalmente a la red eléctrica de la ciudad.

Tales acciones contribuyen a dar forma al entorno bullicioso que caracteriza al centro de la ciudad. Sin embargo, San Nicolás también presenta un sonido muy especial que lo diferencia del resto de actividades comerciales que caracterizan el centro de la ciudad: el sonido de golpes producidos por las guillotinas de papel y las imprentas. Estas máquinas (antiguas impresoras rotativas, tipográficas, cortadoras de papel e impresoras offset) 
generan sonidos muy particulares que juegan un papel importante en la configuración del entorno sonoro del vecindario, imprimiendo un carácter acústico que puede identificarse fácilmente y que lo diferencia de otras áreas del centro de la ciudad. Sin embargo, los sonidos de las imprentas tienden a estar "ocultos por el tráfico pesado y la actividad de la calle", como "el sonido sorprendente de los vendedores ambulantes que recurren a los altavoces para promocionar la última película o álbum de música, el paisaje sonoro está enmascarado por el rugido de las motocicletas, automóviles, camiones y autobuses que funcionan con diésel". (Cuellar y Llorca, 2017). Aunque el sonido de las impresoras es un sonido que caracteriza el entorno sonoro del vecindario, este no es un sonido invasor o dominante. Si las máquinas se escuchan desde la calle, tiene más que ver con el hecho de que las empresas están ubicadas en la parte delantera de las casas, muy cerca de la calle y que la mayoría de estos funcionan con puertas abiertas, por lo que las máquinas son visibles y audibles para los transeúntes. Las horas de trabajo para la mayoría de las empresas son de 8:00 a.m. a 1:00 p.m. y 2:00 p.m. A las 6:00 p.m., cumpliendo con los estándares impuestos a las industrias en los sectores residenciales.

La evolución de la industria de la impresión en Cali creció paralelamente a la ciudad, su desarrollo comercial, su industrialización gradual y su apertura progresiva a los mercados internacionales. Durante los primeros años del siglo XX, la industria en cuestión parecía estar estrechamente vinculada al trabajo de varios periódicos y al trabajo cívico, político y educativo de destacados periodistas locales. El mapeo metodológico diseñado para recopilar información sobre los sonidos del vecindario, así como las narraciones que apuntaron a describir el papel que desempeñan en la configuración de su entorno acústico, revelan cómo un análisis más cercano de los paisajes sonoros de la ciudad puede constituir rutas alternativas para comprender las relaciones que coexisten entre individuos. y su entorno.

\section{Coyoacán}

Coyoacán fue una población que se incorporó en 1929 al Distrito Federal, lo que modificó su actividad productiva agrícola y manufacturera anterior a una comercial y cultural, de enorme atractivo turístico. Un viajero lo dice: Coyoacán es casi una continuación de San Ángel, pero allí hay más árboles y cada casa tiene su jardín o su huerto lleno de naranjos. Las dos villas sirven como una casa de campo para los ricos en la Ciudad de México. (Wright, 1987).

Coyoacán se mantuvo como un lugar de descanso en las afueras de lo que era la ciudad con una sola conexión, la Calle Real (ahora Francisco Sosa). Es durante la primera mitad del siglo $\mathrm{XX}$ que experimenta importantes transformaciones, inaugurando en 1926 la carretera México - Coyoacán, antiguo Antiguo Camino de Cortés. En la década de 1930, todavía estaba formada por haciendas, extensas áreas ejidales e innumerables ranchos y aldeas que le dieron un aspecto semirural, donde la presencia de campesinos era incuestionable. Arroyos y pozos, extensas áreas ajardinadas y un gran número de construcciones arquitectónicas de enorme riqueza histórico-cultural fueron los elementos que le dieron en 1934 el primer decreto de patrimonio, sin embargo, en la próxima década, el desarrollo urbano sería apoyado por la división de haciendas y la apertura de nuevas avenidas y calles, gracias a los nuevos sistemas de transporte. Por lo tanto, se incrementó la accesibilidad a esta zona de la ciudad, lo que permitió la llegada de nuevos residentes en busca de "paz y tranquilidad". Inicialmente, el área se consolidó mediante el establecimiento de casas de campo para las familias acomodadas de la Ciudad de México y más tarde, a partir de 1890, con la conformación de la Colonia del Carmen, que alberga el Jardín Hidalgo y el Jardín Centenario.

El crecimiento poblacional del municipio aumenta con la inauguración de Ciudad Universitaria en 1954. En 1972, una gran parte de su centro fue decretado como área histórica y en 1990 su crecimiento humano, 
urbano y comercial fue declarado Zona Monumento Protegida (Ramírez, P., 2009). El Jardín Hidalgo ha sido, desde el inicio de la Conquista, un espacio público de uso político y un testigo silencioso de los cambios de poder. Lo que hoy es el edificio de la Alcaldía de Coyoacán (poder político actual) fue la sede del primer gobierno de la sede de La Nueva España comandada por Hernán Cortés. Originalmente, Jardín Centenario era parte del atrio de la Iglesia de San Juan Bautista y su acceso era por los arcos que aún se encuentran al final de la calle Francisco Sosa. Al introducir el tranvía a Coyoacán se divide con la línea de la calle Felipe Carrillo Puerto, y se dividen en dos plazas: Jardín Hidalgo y Jardín Centenario, destinadas a la actividad comercial.

En nuestra memoria, Coyoacán sigue siendo un lugar que reúne a intelectuales y artistas notables que construyeron y determinaron la cultura de la ciudad. Novo y García describen el paisaje tranquilo y casi enclaustrado del vecindario que conserva la imagen de una ciudad "colonial" en el centro de Coyoacán (Novo, 1986). El área está llena de calles estrechas y empedradas y pequeñas plazas, que se diseñaron durante el período colonial que le dan al área una identidad distinta y bohemia. El área está llena de casas en su mayoría unifamiliares, que fueron antiguas mansiones, y casas de campo construidas entre los períodos coloniales hasta mediados del siglo XX.

El turismo ha sido una bendición mixta para el centro histórico ya que los establecimientos comerciales se abren, ayudando a la economía, pero también expulsando a los residentes; mientras que las empresas en crecimiento favorecen a la economía, los grupos residentes temen que el área pierda su carácter, dado que el crecimiento comercial ha contribuido a intensificar los decibeles en el área, lo que a su vez tiene un gran impacto en el entorno sonoro. Los grupos de vecinos se han formado para enfrentar los cambios y preservar el valor histórico del área, ya que esta parte de la ciudad ha sido tradicionalmente tranquila y pacífica; compuestas por casas grandes, antiguamente utilizadas para el descanso, y rodeadas de grandes jardines que las protegen de la actividad de la vida pública en contraste con lo que sucede en sus dos plazas principales, donde estas casas contrastan con los pocos edificios de uso mixto en altura que existen en el zona.

Jardín Centenario alberga una diversidad de sonidos (entre 54 y $69 \mathrm{~dB}$ ), donde el sonido que predomina es el del agua corriente que emana de la emblemática fuente de los coyotes. El murmullo y la risa de las personas que visitan la plaza también son sonidos constantes, al igual que el ruido del tráfico que viene de la calle Felipe Carrillo Puerto. En ciertos momentos del día, también se puede escuchar el silbato de los policías que dirigen el tráfico, así como el toque de las campanas de la parroquia de San Juan Bautista (1552) que anuncian misa y otros eventos. La plaza está rodeada de restaurantes y otros locales comerciales, y hay músicos alrededor de la zona en busca de turistas que puedan contratarlos para una serenata privada a cambio de un par de dólares. Los organilleros son una característica común en el entorno sonoro de la plaza, al igual que los sonidos de los móviles y los juguetes que funcionan con el viento y que frecuentemente se ofrecen a la venta. El canto de los pájaros es muy fuerte en ciertas partes de la plaza, en su mayoría procedentes de los árboles que rodean el jardín. Entre los otros sonidos que se escuchan con mayor frecuencia se encuentran la explosión de cuetes en las múltiples fiestas parroquiales y las generadas por motocicletas, automóviles e incluso aviones, siendo el último lo suficientemente poderoso como para alterar los zumbidos continuos que sirven como sonido de fondo.

Los visitantes de Coyoacán — desde dentro de la ciudad y extranjeros - se sienten atraídos por la configuración urbana de sus calles y plazas enmarcadas por grandes muros de piedra, que lo hacen incomparable con otras partes de la ciudad; hay otros que van a las plazas antes o después de visitar la casa de Frida Kahlo, que está a pocas cuadras de 
distancia. El consumo cultural del área, que recibe miles de visitantes los fines de semana (se estima que es la segunda área más visitada después del Zócalo) es un problema para los residentes del área que han visto gradualmente los cambios en su vecindario.

El entorno sonoro responde a la actividad comercial que tiene lugar en Jardín Centenario; rodeado de restaurantes y bares que hacen que las voces y las conversaciones se fusionen con el sonido de las bandejas y los platos, la risa y la música de diferentes géneros atraídos por los visitantes que se unen y dan un sonido muy especial.

El sonido es representativo de los encuentros y desacuerdos, de las relaciones que se acercan y de los conflictos que surgen entre los residentes y los visitantes, que se presentan a través de las numerosas quejas que recibe la Procuraduría Ambiental y de Ordenamiento Territorial (PAOT). En este sentido, este espacio definido como agradable en términos de sonido, podría considerarse como un espacio imperfecto, como lo sugiere Jaime Lerner. En este tipo de espacios, "se permite la coexistencia de culturas, costumbres y diversas economías" (Lerner en El País, junio de 2018), lo que nos lleva a preguntarnos si es necesario redefinir el concepto de "buen ambiente sonoro".

\section{Conclusiones}

El sonido se ha convertido en un tema recurrente de investigación en el campo del urbanismo. La contaminación acústica se ha incluido recientemente en las agendas gubernamentales debido quizás al crecimiento sustancial de la población urbana y los desafíos que surgen de esto. La mayoría de los estudios sobre sonido, principalmente en contextos urbanos, se han centrado en el control del ruido, en particular en el espacio público, fomentando una serie de cálculos y mediciones de entornos sonoros, así como en la mejora del control del tráfico, la insonorización de edificios y otras regulaciones acústicas (García, 2001). Sin embargo, las implicaciones del sonido en la vida cotidiana tienen un matiz más complejo en el que deben considerarse las condiciones subjetivas de la percepción, así como factores ambientales, físicos y sociales que determinan que la recepción de un estímulo acústico sea agradable o molesta. Es importante enfatizar el dinamismo del sonido, que permite el enriquecimiento y el significado de diferentes lugares en los que se desarrolla la vida; aunque, desde una evaluación subjetiva del entorno auditivo, esto depende no solo de su intensidad sino también de la información que transmite, el contexto en el que se percibe y los significados sociales y culturales que le atribuye el receptor (De Gortari, 2010).

En los casos analizados queda claro que los sonidos grabados son inherentes a la actividad $\mathrm{y}$ al reconocimiento de los espacios como lugar. En este artículo se reportan dos casos de estudio de áreas con actividad intensa, laboral y comercial, ambos considerados ruidosos. Los paisajes sonoros urbanos están compuestos principalmente por una serie de estímulos acústicos que exceden los límites sugeridos por la Organización Mundial de la Salud (OMS). El ruido se ha convertido en un elemento característico de la vivienda urbana, y como señala Kreutzfeldt, "no sería un entorno de sonido urbano si el ruido nunca se escuchara" (Kreutzfeldt, 2010). En este trabajo hemos tratado de mostrar que el sonido, en esos espacios de intercambio, es ruidoso, pero también, como en el caso de San Nicolás, son fuentes que ofrecen información sobre la historia del barrio. Es necesario, entonces, repensar la evaluación de sonido de los espacios urbanos y sus efectos en la calidad de vida de las personas, entendiendo el sonido no solo como una señal a procesar, sino como información a analizar, lo que ha permitido otros enfoques. Como lo sugiere Lankford, E. (2009), el ruido es idiosincrásico, es una mera interpretación de un sonido escuchado. El objetivo es mostrar cómo, a través de la experiencia del espacio ejemplificado a través de lo que escuchas, tienes información con la que trabajar más estrechamente con el lugar y con los que lo habitan.

En este trabajo se considera que un espacio saludable también implica un espacio de actividad: San Nicolás como espacio de trabajo y Coyoacán como recreativo. Para mejorar - construir - evaluar - revalorizar el entorno acústico requiere una construcción cívica que relacione y defina esa identidad, 
que pase por acuerdos en los que la comunidad participa en la construcción de su entorno en busca de mejorar la calidad de vida en términos adecuados. Entendemos "el espacio y el paisaje como una entidad viviente" (Llorca, 2016), donde el espacio "incluye lo físico y lo social como un conjunto indisoluble de sistemas de objetos y sistemas de acciones" (Santos, 2000 en Llorca, 2016 ). Es necesario establecer e identificar zonas acústicas sensibles para proponer oportunidades de acción a corto, mediano y largo plazo, invitando a la comunidad a participar activamente en la disminución de los efectos del ruido, mientras se trabaja en recomendaciones de políticas públicas sobre asuntos sólidos. La estrategia de proyectos como estos ayuda a comprender la complejidad de las relaciones de un entorno y luego avanzar y poder tomar decisiones sobre qué partes pueden conducir a la intervención y las acciones para transformar la realidad sólida en un sentido positivo. El propósito es comenzar a desarrollar herramientas de trabajo en este campo para determinar, por ejemplo, de qué manera influye un entorno construido en el entorno sensible, y es capaz de generar modificaciones que conduzcan a una mejora del aspecto positivo de nuestro paisaje sonoro. Mejorar la calidad de vida en la ciudad implica, entre otros factores, una administración positiva del paisaje sonoro, para lo cual es necesario ampliar el horizonte de la lucha contra el ruido. La investigación de paisajes sonoros representa este cambio de paradigma, ya que implica no solo medidas físicas sino también la cooperación de las ciencias humanas y sociales para explicar la diversidad de paisajes sonoros en todos los países y culturas, y considera los sonidos ambientales como un "recurso" en lugar de un "desperdicio" (Brown , 2010, 29). Esto se logra a través de una evaluación subjetiva del paisaje sonoro que no solo depende de la intensidad sino también de la información que contiene, los contextos percibidos, así como las significaciones sociales y culturales que el receptor le atribuye. Las cartografías sonoras y la metodología de trabajo que surgen de estas investigaciones dan cuenta de las posibilidades que se abren al cruzar el mapa físico del espacio y continúan incorporando otros aspectos del lugar que desempeñan un papel importante en las construcciones sociales y culturales. Con lo que damos sentido a estos espacios, los mapas utilizados en este trabajo muestran que esta información es una base para ejemplificar la experiencia del lugar, no podemos pensar en mejorar la salud urbana de la ciudad sin complementarla con la forma en que se vive el espacio.

Las cartografías sonoras, cuando se superponen con información sobre cómo se percibe el entorno sonoro, reflejan la organización espacial de la ciudad y la experiencia de la vida en la ciudad. De este estudio integral se podrían derivar planes de acción profundamente vinculados a las necesidades de los habitantes a través de planes estratégicos relacionados con el diseño y la rehabilitación de horarios sólidos en un sector estudiado en la ciudad. Esto demostraría que podría haber otras alternativas para enfrentar el problema de la contaminación acústica.

\section{Referencias}

Amphoux, Pascal, (2001) Le temps du paysage sonore, quelques critère d'analyse, Cresson,

Alonso, M. (2003). El entorno sonoro. Un ensayo sobre el estudio del sonido medioambiental. Recovered from https:// www.academia.edu/197497/E1_entorno_ sonoro._Un_ensayo_sobre_el_estudio_ del_sonido_medioambiental_The_Sonic_ Environment._An_Essay_About_the Study_of_Environmental_Sound_

Atienza, R. (2008). Identidad sonora urbana: tiempo, sonido $\mathrm{y}$ proyecto urbano. Recovered from http://www.cresson.archi. fr/EQ/Identidad\%20sonora\%20urbana.pdf

Attali, J. (1985). Noise: The Political Economy of Music. Manchester, UK: Manchester University Press.

Augoyard, J. (1997). La sonorización antropológica del lugar. In M. Amerlinck, M. (Ed.), Hacia una antropología arquitectónica (pp. 205-219) Guadalajara, México: Universidad de Guadalajara.

Berglund B, et al, Guidelines for community noise,

www.vho.int/docstore/peh/noi- se/guidelines2. html (consulta 20 febrero 2007)

Blesser, B. (2007). Spaces Speak Are you 
listening?, MIT press.

Brown, L. 2010. Soundscapes and environmental noise management. Noise Control Engineering Journal, 58 (5) Sept- Oct 2010. A review of progress in soundscapes and an approach to soundscape planning,

Carles, J and Palmese, C. (2009). "Procesos del cambio en el paisaje sonoro urbano. Nuevos desarrollos metodológicos." Foro Mundial de Ecología Acústica. Megalópolis sonoras. Identidad cultural y sonidos en peligro de extinción. Conaculta, Fonoteca Nacional, México

De Gortari, J. (2010). "La revalorización de los sonidos y la calidad Sonora ambiental del Barrio Gótico, Barcelona", Doctoral Dissertation, Departartamento de Construccions Arquitectòniques i, Universitat Politècnica de Catalunya.

De Gortari, J. (2013). Guía Sonora para una ciudad, UAM-Juan Pablos, México.

Krausse, Bernard, (1993) The Niche Hypothesis: a virtual symphony of animal sounds, the origins of musical expression and the health of habitats, Soundscape Newsletter, World Forum for Acoustic Ecology, Simon Fraser Univ., June, 1993.

García Rodríguez, Amando, (2001) Environmental urban noise, WITpress, Gran Bretaña.

Hellstrom, Björn (2001) Modelling of Sounds in public spaces, Conference paper: Sound Prac- tice, College of Arts, Totnes, England, febrero 2001.

Jacobs, J. (1961). The death and lif of great American cities, Random House.

Kang, J. 2006. Urban sound environment. Taylor and Francis, London.

Lankford, E. (2009) Urban Soundscapes as Indicators of Urban Health, Environment, Space, Place, Vol. 1, No. 2 (Fall 2009), pp. 27-50López, et.al, 1997,

Llorca, J. (2016). Paisaje sonoro y territorio. El caso del barrio San Nicolás en Cali, Colombia. Revista INVI, 32(89).

Mumford, L. (1938) The City in History: Its Origins, Its Transformations, and Its Prospects

Pelinski, R. (2012). El oído alerta: modos de escuchar el entorno sonoro. Recuperado de http://cvc.cervantes.es/artes/paisajes sonoros/p_sonoros $01 /$ pelinski/pelinski_01. $\mathrm{htm}$

Ramírez Kuri, P. (2011) La reinvención del espacio público en la ciudad fragmentada, UNAM.

Raimbault, M. and Dubois, D. 2005. Urban soundscapes: experiences and knowledge, Cities, 22, 339-350.

Rice, T. (2008). "Beautiful murmurs": Stethoscopic listening and acoustic objectification. Senses \& Society, 3 (3), 293 306.

Schafer, M. (1977). Soundscape: Our Sonic Environment and the Tuning of the World. Rochester, USA: Destiny Books.

Schafer, R. M. The tuning of the world

Southworth, M. (1969) The sonic environment of cities, Environment and Behavior 1(1) January 1969.

Thibaud, J. (2003) L'ambiance comme expression de la vie urbaine. Cosmopolitiques. Cahiers théoriques pour l'écologie politique, Editions Apogée/ Cosmopolitique, 2004, pp. 102 - 113.

Truax, B. 1978, Handbook for acoustic ecology, Cambridge Street Publishing.

Westerkamp, H. (1972). "Listening and Sound Making: a Study of Music-as-Environment", Master's Thesis, Simon Fraser University. 